\title{
Coulisses
}

Revue de théâtre

4 | Été 1991

Varia

\section{Le Théâtre de Stanislavski}

\section{Françoise Lesourd}

\section{(2) OpenEdition}

Journals

Édition électronique

URL : http://journals.openedition.org/coulisses/1689

DOI : $10.4000 /$ coulisses.1689

ISSN : 2546-9460

\section{Éditeur}

Presses universitaires de Franche-Comté

\section{Édition imprimée}

Date de publication : 1 juin 1991

Pagination : 41-51

ISSN : 1150-594X

\section{Référence électronique}

Françoise Lesourd, «Le Théâtre de Stanislavski », Coulisses [En ligne], 4 | Été 1991, mis en ligne le 04 juillet 2017, consulté le 22 octobre 2019. URL : http://journals.openedition.org/coulisses/1689 ; DOI 10.4000/coulisses.1689

Ce document a été généré automatiquement le 22 octobre 2019

Coulisses 


\title{
Le Théâtre de Stanislavski
}

\author{
Françoise Lesourd
}

1 Le milieu culturel moscovite, au tournant du siècle, opposait un certain esprit de liberté et de non-conformisme au caractère plus compassé de l'autre capitale, Pétersbourg, ville de l'aristocratie et de la haute administration. Leur prospérité laissait à de nombreux industriels et commerçants (plus fréquemment installés à Moscou) d'importants loisirs. Le mécénat était un phénomène plutôt moscovite.

2 Constantin Stanislavski, né en 1863 dans une famille d'industriels, les Alexiev (fabriquants de brocart), est l'émanation de ce milieu. La famille Alexiev réservait au théâtre tous ses moments de liberté - un théâtre de type familial qui tenait une place importante dans la vie mondaine de l'époque en Russie. Pour les enfants Alexiev, l'été se passait à monter des spectacles, à répéter et à jouer devant parents et amis.

3 Il ne s'agissait pourtant que de loisirs: lorsque le futur Stanislavski décide de se consacrer au théâtre de manière semi-professionnelle, il est tellement incertain quant à la réaction paternelle qu'il prend, pour l'affiche, ce nom d'emprunt qu'il gardera toute sa vie - hommage à un acteur-amateur particulièrement admiré.

4 Entre-temps, lors de voyages à Paris, il avait constaté que l'enseignement du Conservatoire, trop traditionnel, lui convenait mal, alors qu'il se sentait plus attiré par le théâtre de boulevard. 


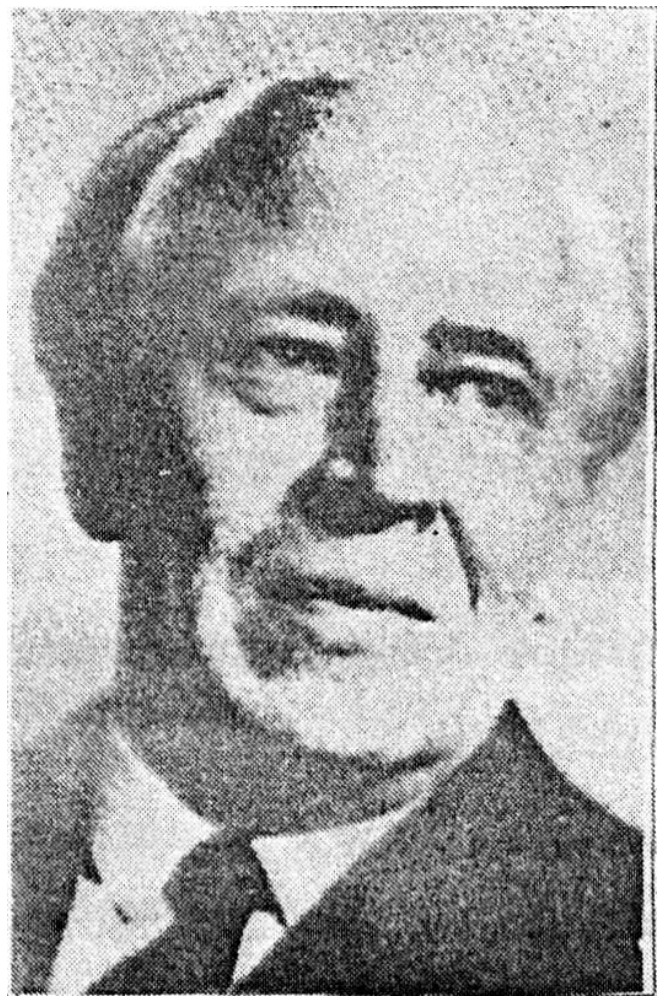

K. C. Stanislavski.

5 Ses véritables débuts se situent en 1887 , avec la création d'une "Société d'art et de littérature ", qui comprend, à ses côtés, un acteur professionnel déjà relativement célèbre, Fédotov, un professeur de chant, et le peintre Korovine, un de ceux qui participeront au renouveau pictural des années 1900-1910, en particulier dans les décors de théâtre. La "Société d'art et de littérature » comportait un cercle dramatique grâce auquel, tout en recevant une formation d'acteur, Stanislavski et ses amis commençaient à se produire occasionnellement sur de petites scènes (essentiellement dans des clubs). Dès ce moment, leurs spectacles révèlent un esprit de recherche et une volonté de renouveler la création théâtrale.

6 L'école naturaliste avait commencé à bousculer les conventions du théâtre académique. Le symbolisme, cherchant des moyens d'expression nouveaux, avait encore exacerbé cette tendance.

7 La troupe des Meininger, en Allemagne, s'était rendue célèbre dès les années 70 par ses recherches d'authenticité dans la reconstitution historique des costumes et des décors. Elle fondait son travail sur quelques principes qui, à cette époque, révolutionnaient l'art de la mise en scène : au nom du naturel, elle évitait le « jeu frontal », les acteurs n'étant plus tenus de déclamer face au public. Lorsque la vraisemblance de la situation l'exigeait, certaines tirades pouvaient être prononcées dos aux spectateurs. La théorie de la « quatrième paroi », posant l'existence d'une séparation invisible mais absolue entre la scène et les spectateurs, devait également contribuer à cet effet de naturel. Enfin, la figuration au sens strict était abandonnée : aucun rôle, même minime, même à peine perceptible pour le public, ne devait être interprété de façon passive.

8 Ces nouvelles tendances de la mise en scène, qui ne se limitèrent pas aux Meininger (en France, elles furent suivies par le metteur en scène Antoine), suscitèrent l'intérêt du cercle de Stanislavski. Le théâtre de la «Société d'art et de littérature " se passionne 
aussi pour les effets de réel. Leur Chevalier avare, de Pouchkine, qui reconstitue dans les moindres détails une atmosphère médiévale, est un premier succès. Stanislavski, pour mieux se pénétrer de son rôle, s'était fait enfermer dans les souterrains d'un vieux château, où, un moment, il s'était cru oublié pour toujours !

Dès l'abord, Stanislavski est classé par les critiques dans la catégorie des acteurs « de genre ", c'est-à-dire destinés à des emplois très typés, surtout sociologiquement. Cette recherche très poussée de l'exactitude documentaire vivifiante parce qu'anticonventionnelle, rencontre aussi, assez rapidement, ses limites : elles seront sensibles, en particulier, dans les mises en scène de Shakespeare. En dépit de toutes les études documentaires qui les auront précédées (pour Jules César, on était allé à Rome étudier et reconstituer tous les accessoires, y compris la texture des tissus antiques pour les costumes), certaines mises en scène manquent de force dramatique. Stanislavski le reconnaît : « Nous étions inférieurs à nos décors. »

10 Son inventivité pratique lui permet, au contraire, de réussir dans les pièces fantastiques - La Cloche engloutie de Hauptmann, par exemple. Sur la scène russe, cette recherche d'authenticité, l'intimité du décor, le naturel du jeu, apparaissent comme des phénomènes novateurs, bien que la troupe de Stanislavski s'affirme aussi comme l'héritière des traditions du Théâtre Maly de Moscou - celles de la vérité psychologique.

11 Le cercle de Stanislavski continuait à se produire sur des scènes de fortune. Jusqu'en 1902, il ne possède pas de salle de spectacle qui lui soit propre. En 1897, pourtant, la rencontre de Némirovitch-Dantchenko, dramaturge et critique théâtral, qui va très vite devenir le conseiller et l'administrateur de Stanislavski, aboutit à la création d'une troupe régulière, une véritable compagnie théâtrale. Ce nouveau théâtre (MKHAT: "Théâtre artistique de Moscou ») se proclame " accessible à tous " (" obscedostupnyj »). Stanislavski le veut ouvert à un public populaire. Mais ses préoccupations sociales et son caractère novateur lui attirent la méfiance du Conseil Municipal de Moscou, et les subventions espérées sont finalement refusées. Le financement de la nouvelle troupe se fera sur fonds privés, et même un peu plus tard grâce au mécénat, celui de l'industriel Morozov.

12 La deuxième rencontre décisive - entre Stanislavski et Tchékov - est également due à Némirovitch-Dantchenko. Il avait assisté, en 1896, à la première représentation de $L a$ Mouette, au Théâtre Alexandre de Pétersbourg, qui avait été l'un des «fours » les plus mémorables dans l'histoire du théâtre russe, malgré la participation d'une grande actrice de type « lyrique » (selon l'expression d'Alexandre Blok), Vera Komissarjevskaïa dans le rôle de Nina. Par une sorte de malentendu, la pièce avait été présentée à un public petit- bourgeois pour l'anniversaire d'une actrice comique. On s'attendait à un vaudeville, et les spectateurs, déçus, réagirent par un scandale.

13 Persuadé de la valeur de cette pièce, Némirovitch-Dantchenko se heurte d'abord à la résistance de Tchékov lorsqu'il lui propose de faire jouer La Mouette à Moscou : "Je ne présenterai pas ma pièce à Moscou. Plus jamais je n'écrirai pour le théâtre... » Finalement, le MKHAT est autorisé à renouveler l'expérience.

14 En décembre 1898, c'est la première représentation à Moscou par la troupe de Stanislavski. Elle interprète là sa première oeuvre contemporaine, dont l'originalité repose sur l'absence de situations pathétiques traditionnelles et sur l'inaction apparente des personnages. Pendant les répétitions, Tchékov n'avait cessé d'insister sur la simplicité et l'intériorisation du jeu, et sur l'importance des silences. 

mort, l'activité de Tchékov, auteur dramatique, n'est pas limitée à la fin de sa vie. Dès ses années de lycée, à Taganrog, il écrivait pour le théâtre et organisait des spectacles. Dans les débuts de sa carrière littéraire, les mêmes sujets revenaient parfois dans ses nouvelles et ses pièces - courtes (la plupart du temps en un acte), comiques, reposant sur une intrigue simple dont les ressorts appartiennent à la tradition du vaudeville (misogynie, goût du lucre, moeurs de la petite-bourgeoisie...). Dans les pièces comme dans les nouvelles, à cette époque, il recherche le succès facile, la vivacité, la légèreté.

Avant 1896 cependant, trois pièces se détachent sur cet arrière-plan, à la fois par leur dimension et leur inspiration, qui annonce les "grandes" pièces de la fin. Ce sont: Ivanov, Platonov, L'Homme des bois. Mais, de l'avis même de Tchékov, elles en sont encore au stade de l'expérimentation. Par rapport aux nouvelles, l'auteur leur accorde peu d'importance. Mais elles montrent la place que tient la création théâtrale pour Tchékov, et les tâtonnements qui ont précédé l'élaboration des quatre pièces célèbres.

Malgré les succès, la coopération entre Tchékov et Stanislavski s'est révélée dès le début très difficile. Le premier désaccord porte sur le personnage de Trigorine, dans $L a$ Mouette, interprété par Stanislavski. À son propos, Tchékov écrit dans une lettre à Gorki : «Trigorine... traverse la scène, et parle, comme un paralytique ; il est, certes, "privé de volonté ", mais l'interprète a compris cela de telle façon qu'on a mal au cœur à le regarder ». Sa déconvenue était si forte qu'il aura du mal à croire, par la suite, que Stanislavski puisse être un excellent interprète d'Astrov dans Oncle Vania.

La froideur relative de Tchékov, qui étonne Stanislavski lors de leur première rencontre après la première de La Mouette, n'est donc pas seulement due, vraisemblablement, à « son naturel peu expansif » (« Anton Pavlovitch me serra la main plus fort qu'à l'accoutumée, il eut un bon sourire... et c'est tout »).

Un des principaux mérites de Stanislavski est l'invention du « paysage auditif. Au début de La Mouette, il doit créer l'impression du silence - ce qui est impossible au théâtre par la simple absence de bruit. Il imagine alors de la suggérer en faisant entendre le chant des grenouilles. Le succès même de ces trouvailles comportait pourtant un risque. En devenant trop systématiques et répétitifs, ces procédés lui attireront encore une fois les critiques de Tchékov. Les problèmes de bruitage deviennent vite une source de disputes tragi-comiques entre l'auteur et son metteur en scène. Dans une lettre à Olga Knipper (sa femme, qui était une actrice du MKHAT), au sujet de La Cerisaie, Tchékov se plaint : "Constantin Serguéiévitch veut, au deuxième acte, faire passer un train, mais à mon avis, il faut l'en dissuader. Il voudrait aussi des grenouilles, et puis des poules d'eau... ! » Le ton de certaines lettres à Stanislavski est révélateur: "Cher Constantin Serguéiévitch, la fenaison a lieu d'habitude vers les 20-25 juin; or à ce moment, il me semble bien que les poules d'eau ne chantent plus, de même que les grenouilles. Le seul que l'on entende encore crier, c'est le loriot... »

Les effets de lumière étaient, eux aussi, une des grandes découvertes de Stanislavski : le feu qui brûle dans la cheminée, la scène éclairée par la seule flamme d'une bougie... Mais il reconnaît lui-même qu'il avait tendance à en abuser dans sa volonté de stimuler la «mémoire affective ». Excédé, Tchékov avait parlé un jour d'écrire une pièce qui commencerait par ces mots: "Comme on est bien, quel silence! On n'entend ni oiseaux, ni chiens, ni coucou, ni chouette, ni rossignol, ni horloge, ni clochettes, ni même le moindre grillon... » 
21 Ses didascalies étaient d'une précision, d'un laconisme et d'une intransigeance redoutables. Au premier acte de La Cerisaie, il insiste pour que Charlotte soit accompagnée d'un petit chien "hirsute, à moitié crevé, aux yeux chassieux...» $\mathrm{Au}$ deuxième acte, il demande un paysage à l'arrière-plan duquel "on peut distinguer la ville par beau temps..." Trigorine porte "un pantalon à carreaux et des chaussures percées", et Oncle Vania une cravate de soie : aux yeux de Tchékov, ces indications doivent suggérer à elles seules toute l'interprétation de ces deux personnages.

La première de La Mouette, quoi qu'il en soit, fut un triomphe, malgré les inquiétudes de Stanislavski, angoissé de monter une pièce aussi novatrice, où le véritable drame n'est pas dans l'action mais "dans l'inaction des personnages", et conscient de sa responsabilité à l'égard de l'auteur, déjà très gravement malade à l'époque et traumatisé par son premier échec. De ce jour, l'image stylisée d'une mouette devint l'emblème du théâtre. Elle n'a jamais cessé, depuis, de figurer sur le rideau de scène et les programmes.

Le MKHAT devient ainsi le théâtre attitré de Tchékov. Ses liens avec la troupe de Stanislavski sont tellement étroits qu'il lui arrive, en particulier dans La Cerisaie, de modifier certaines répliques en fonction des acteurs. En 1899, ils mettent en scène une deuxième pièce de Tchékov, Oncle Vania. À son propos, l'auteur emploie pour la première fois le terme d' « atmosphère », qui sera repris par Meyerhold et deviendra la principale définition du théâtre tchékovien. Paradoxalement, c'est la visée naturaliste qui favorisa la naissance d'un tel théâtre. L'intention de Stanislavski - remplir l'espace scénique d'objets familiers - contribua à la création de cette "atmosphère ", mais à condition de rester suffisamment discrète. D’où le souci constant de Tchékov (qui correspond d'ailleurs à sa méthode narrative): trouver le petit détail concret significatif, mais sans écraser la représentation sous l'abondance des objets. Il faut bien reconnaître que les mises en scène actuelles du MKHAT semblent confirmer les inquiétudes de Tchékov : la reconstitution scrupuleuse, pédante, d'un environnement véridique dans les moindres détails, contribue à figer les impressions et à exclure précisément cette notion d'atmosphère.

Dans Oncle Vania, Stanislavski interprète Astrov, et Tchékov lui suggère seulement de jouer tout ce rôle " en sifflotant » - ce qui prend tout son sens dans une lettre à Olga Knipper du 2 janvier 1901 : «Les gens qui, depuis longtemps, portent en eux le malheur et s'y sont habitués, se contentent de siffloter, et prennent souvent l'air pensif. »Ce rôle fut l'un des meilleurs de Stanislavski, dont le jeu était tout en nuances.

Pour Les Trois Sœurs, l'année suivante, le succès est moins éclatant : l'effet de surprise est passé ; 1905 approche et l'atmosphère sociale se modifie. Le pessimisme fin de siècle, l'humeur "décadente" suscitent moins d'échos. L'action politique apparaît de nouveau possible et souhaitable.

Sensible à cette modification du climat social et lassé par ce qu'il considère comme un manque d'énergie dans le jeu de Stanislavski, Tchékov décide alors d'écrire quelque chose de vif, d'alerte, une "vraie comédie ", selon ses propres termes. Il affirme, en mars 1901 : «Ma prochaine pièce doit absolument être drôle, très drôle, au moins dans sa conception d'ensemble. » À un autre correspondant il confie : "Je rêve d'écrire une pièce si drôle que le diable lui-même en perdrait son latin... » Cette pièce « drôle », c'est La Cerisaie. 

bien qu'il dure encore. Peut-on jouer La Cerisaie, avec sa coloration élégiaque si marquée, comme une pièce "gaie "? Peu de metteurs en scène (à part peut-être Strehler, en accentuant le côté cabotin de Ranevskaîa) ont relevé cette gageure, et surtout pas Stanislavski. Pourtant, dès les premières répétitions, Tchékov s'insurge : «Pourquoi me dis-tu (il écrit à Némirovitch-Dantchenko)... que ma pièce est pleine de gens qui pleurent ? Où sont-ils donc? La seule qui pleure, c'est Varia, et encore, pour la seule raison qu'elle est pleurnicheuse par nature. Ses larmes ne doivent susciter aucune tristesse chez les spectateurs... Et il n'y a pas de cimetière au deuxième acte... » Cette dernière phrase montre toute la subtilité des remarques scéniques de Tchékov: il s'agit, selon ses propres termes, d'un "ancien cimetière", dont il ne reste plus que «quelques pierres tombales »... Mais le plus étonnant, c'est que les didascalies de cette pièce comportent, presque à chaque page, «il pleure", "pleurant", "à travers ses larmes "...

Il reproche aux acteurs de mener la pièce sur un rythme languissant: "Un acte qui devrait durer 12 minutes au maximum, vous mettez 40 minutes à le jouer. Tout ce que je peux dire, c'est que Stanislavski m'a bousillé ma pièce... » (Lettre à Olga Knipper). Il proteste énergiquement contre l'emploi du mot «drame» sur les affiches et dans les journaux.

Stanislavski, comme il le dit lui-même, redoutait le rôle de Lopakhine que Tchékov lui réservait: «Il faut avoir une très grande maîtrise du ton pour pouvoir donner au personnage une coloration vécue, quotidienne »... « On dit que je réussis mal dans les rôles de marchands... Il est vrai que Lopakhine est bienveillant, tout en ayant de la force de caractère. Et la Cerisaie, il l'a achetée un peu malgré lui, il en a même eu honte ensuite... »

Il se laissera finalement convaincre de jouer ce rôle. Mais pour le ton général de la pièce, c'est la volonté du metteur en scène qui semble bien l'avoir emporté sur celle de l'auteur, et il s'agit d'un malentendu heureux, puisqu'elle connaît un véritable triomphe.

31 Est-il vraiment possible, d'ailleurs, de la jouer comme une comédie au sens strict? La note lyrique, en tous cas, avait été suggérée par Tchékov lui-même : «Au premier acte, on voit par la fenêtre, des cerisiers en fleurs, tout un jardin blanc. El les dames sont en robes blanches. " (Lettre à Stanislavski). Lorsqu'il écrit à Némirovitch-Dantchenko, " cette pièce, je l'appellerai comédie ", on voit bien que, pour l'auteur lui-même, la définition ne semble pas aller de soi. Peut-être Tchékov, instruit par l'habitude, avait-il peur de voir Stanislavski en faire trop, et perdre ainsi une distance ironique que rendait particulièrement nécessaire la coïncidence entre le destin du verger promis à la destruction et celui de Tchékov au seuil de la mort. On pourrait aussi expliquer son agacement par la crainte de perdre la polysémie, le flottement de sens qu'il s'appliquait à dégager. Après la coloration élégiaque du spectacle de Stanislavski, c'est une note vraiment tragique que Meyerhold imposera aux mises en scène de cette pièce, lui qui voyait dans La Cerisaie « la tragédie de l'Effroi et du Destin ». Tchékov meurt en juillet 1904.

Cette conception du théâtre, envisagé non plus comme un divertissement mais comme la quintessence de l'art et l'objet d'un culte, conception que défend Stanislavski, assure le succès du MKHAT. Lors de sa tournée à Pétersbourg, en 1904, le peintre Doboujinski (un des fondateurs du premier groupe moderniste en peinture russe, le Monde de l'art) 
rappelle, dans ses Souvenirs, l'effet de surprise de ce spectacle, où la musique légère habituellement jouée pendant les entractes était supprimée, où les retardataires n'étaient pas admis, où même les applaudissements pendant la durée de la représentation étaient prohibés. Au lieu de se lever, le rideau de scène s'ouvrait, ce qui donnait à la représentation un caractère intimiste. Mais surtout, l'aspect artificiel des procédés scéniques, en particulier l'emphase de la diction, avait disparu.

Pourtant, Doboujinski remarque l'insuffisance des décors sur le plan de la technique picturale. Il est vrai que le caractère rudimentaire des toiles de fond, par exemple celle d'Oncle Vania (un mur terne et un escalier assez mal peint) surprend aujourd'hui, lorsqu'on regarde les photos des premières représentations, tellement on est habitué à l'idée que les mises en scène de Stanislavski atteignaient à une sorte de perfection matérielle sur tous les plans. Mais pour les peintres pétersbourgeois de ces années-là années de renouveau néo-classique - ces mises en scène manquent d'éclat et de perfection formelle. Stanislavski en est conscient et il obtient bientôt la collaboration d'un peintre appartenant au Monde de l'art : Simov.

À la suite de ce voyage à Pétersbourg, touché par les idées nouvelles, Stanislavski éprouve la nécessité de dépasser l'influence du naturalisme. C'est là qu'il fait la connaissance du poète Alexandre Blok, le chef de file du symbolisme russe. Pour Blok, bien qu'il apprécie en Stanislavski le désintéressement absolu et le culte de l'art, le renouvellement dû au théâtre réaliste est assez limité, les effets de vraisemblance pouvant tomber à leur tour dans la routine.

La mise en scène de La Cerisaie lui laisse une impression de "déjà vu ». Son scepticisme rejoint celui de Stanislavski, pour lequel, de son propre aveu, cette pièce représentait plutôt un point d'aboutissement, un "air connu ", déjà dépassé, même s'il continuait encore à plaire.

D'autre part, Blok admire profondément Stanislavski pour sa vision globale de l'acteurartiste, exprimant la totalité de l'expérience humaine par la «mise en jeu » de sa vie sur la scène. À travers la "formation de l'acteur », ce que visait Stanislavski, et Blok tout aussi bien, c'était la formation de l'homme lui-même, l'« homme nouveau » appelé à transfigurer la vie.

Leurs efforts communs pour monter deux drames symbolistes de Blok, La Rose et la Croix et La Chanson du destin, n'aboutiront pas. Mais 1905 est une année-tournant pour Stanislavski, au cours de laquelle il se rapproche de Meyerhold, plus radicalement moderniste, et envisage le travail en " studio » - structure plus légère, mieux adaptée à un travail expérimental, pédagogique, centré sur la formation de l'acteur. Pourtant, il reste très sceptique sur lui-même et ses propres possibilités de renouvellement. Il écrit à cette époque : «Je suis un incorrigible réaliste, mes recherches en art ne sont que de la coquetterie ; en fait, au-delà de Tchékov, mon chemin s'arrête... »

Et en effet, dans les années suivantes, sans doute encouragé par la modification de l'atmosphère sociale où les luttes politiques passent au premier plan, Stanislavski met en scène deux pièces de Gorki, Les Petits-Bourgeois, et Les Bas-Fonds. Ici, l'inventivité du metteur en scène contribue à étoffer un texte beaucoup moins dense et subtil que celui de Tchékov, et aussi, pour cette raison, moins contraignant. Le projet de Gorki est une peinture sociale accusatrice, celle des déshérités de la grande ville moderne. Toujours poussé par le même souci d'exactitude scrupuleuse, Stanislavski va jusqu'à visiter les asiles de nuit où l'on recueille les clochards. Il éprouve le besoin d'ajouter au texte une foule de détails concrets (c'est là qu'il fera merveille avec ses nouvelles techniques 
d'éclairage), et de préciser dans ses détails le passé des personnages, dont l'acteur doit tenir compte pour se pénétrer du rôle.

Cette recherche est celle d'un théâtre total : la représentation ne se limite pas à l'espace scénique, il convient de faire deviner la vie derrière la scène. Plus tard, lorsqu'il utilisera un plateau tournant (par exemple pour Othello), il tiendra à ce que les acteurs commencent à jouer avant d'être devant les spectateurs, et continuent bien après qu'ils aient cessé d'être aperçus.

D'une façon générale, la première partie de la carrière de Stanislavski (jusque vers 1910) est marquée par la prééminence du metteur en scène sur l'acteur. Les effets de groupe, les tableaux de foule, les inventions pratiques (une vitre couverte de buée, une gaze tendue sur le devant de la scène pour suggérer une atmosphère de rêve), accaparent l'essentiel de son attention.

41 La période de crise qui va suivre, pendant laquelle il se sent prisonnier d'une routine qu'il a lui-même contribué à créer et se heurte aux limites de la mise en scène réaliste, le conduit à recentrer son intérêt, à le reporter sur l'acteur. Sur son travail de metteur en scène, il en vient à écrire : «Je n'aime pas cette activité, et je ne m'y résigne que par nécessité. » L'effet de vérité créé par un spectacle n'est pas seulement produit, pense-til maintenant, par l'exactitude documentaire dans la réalisation des décors: il faut surtout que l'acteur sente une coïncidence profonde entre son «moi » et le rôle qu'il joue. Cette manifestation authentique de l'«homme intérieur » est ce qui emporte l'adhésion du spectateur.

42 De plus, l'inspiration caractéristique de cette époque où l'on pressentait l'approche d'une révolution, et sa nécessité, est sensible dans les crises traversées par Stanislavski lorsqu'il écrit : « ... il faut apprendre à représenter la vie au théâtre, non pas telle que la perçoit la conscience quotidienne, mais telle qu'elle apparaît dans les moments de plus grande lucidité spirituelle... » Cette lucidité d'ordre supérieur découvre un «homme nouveau ", gage du renouvellement de la vie.

Le travail sur l'acteur est donc un travail sur l'homme lui-même: cette prise de conscience inaugure une nouvelle période de réflexion, au cours de laquelle il se concentre sur le travail de l'acteur et sur sa propre activité de pédagogue. Par deux fois au cours de sa vie, et chaque fois pour des raisons de renouvellement, il se tournera vers le travail en studio - la seconde fois au cours des années 20, mais alors, de plus, sous la contrainte de la pénurie et de la censure.

Sans vraiment abandonner la notion de "réalisme», puisqu'il parle de "réalisme psychologique ", c'est l'époque (1910-1917) où il commence à élaborer son système de formation de l'acteur. L'unité psycho-physique de l'individu est le présupposé qui se trouve à la base de ce "système ». Grâce à elle, chaque attitude est signifiante et, à l'inverse, correspond à une disposition intérieure qu'il convient de préciser pour que cette attitude soit comprise par le public conformément à la volonté de l'acteur et du metteur en scène. Le "paysage sonore ", les effets de lumière et toutes les inventions concrètes de Stanislavski ont ce même but: stimuler la "mémoire affective » du spectateur mais, avant lui, de l'acteur, afin de créer la disposition intérieure nécessaire pour donner au jeu son maximum de crédibilité et tout son pouvoir de révélation.

Si l'ensemble de la mise en scène répond à une grande idée directrice (un «projet d'ensemble»), chaque action envisagée séparément doit aussi comporter son intentionalité propre : l'immobilité même n'est ni une passivité, ni une absence, et sa 
résonance dans la sensibilité du public dépend de la disposition intérieure qu'elle exprime. Les interrelations entre cette disposition intérieure et les effets visibles du comportement peuvent faire l'objet d'une connaissance presque exacte, d'une sorte de science, et Stanislavski consacrera une grande partie de sa vie à élaborer une sorte de théorie du comportement appliquée à l'art du théâtre.

En 1917 et immédiatement après, sa position est ambiguë. La révolution et ses implications culturelles correspondent bien à ses préoccupations sociales, à son rêve ancien de créer pour un public populaire, habituellement éloigné du théâtre. Mais les difficultés matérielles s'accumulent et rendent le travail impossible dans des conditions normales: l'absence de chauffage oblige les acteurs à répéter en manteau. Les restrictions en matière de logement, ajoutées à toutes les autres manifestations de la pénurie, conduisent Stanislavski à établir son théâtre dans son propre appartement. C'est ainsi que, pendant les années les plus dures qui suivent la révolution, sa troupe continue à subsister.

Lorsque la révolution éclate, c'est un artiste déjà pleinement reconnu. Son théâtre est une sorte d'institution dans la vie culturelle russe. Mais au cours des années 20, les tendances plus radicalement novatrices vont l'éclipser. Considéré comme un "réaliste bourgeois", étranger aux abstractions, il est dépassé par ceux qui se proclament révolutionnaires en art - par le théâtre constructiviste de Meyerhold, Taïrov...

Toujours préoccupé d'action immédiate sur la sensibilité du public, et convaincu par là d'opérer dans les mentalités un changement en profondeur bien que difficilement mesurable, il est très loin du théâtre à thèse. Un propos politique au sens strict l'intéresse moins qu'une transformation à long terme de l'« homme » - qui est déjà, en soi, une notion très critiquée à l'époque. Sa forme de sensibilité, caractéristique de l'intelligentsia russe au début du siècle, ne l'incite pas à la sympathie pour la violence révolutionnaire. Sans rupture visible avec le nouveau régime, il demeure cependant un peu en retrait.

Paradoxalement, l'absence de recherches ouvertement révolutionnaires ou de tendances "prolétariennes » (en plus de sa très grande gloire passée) le protégeront pendant les années du stalinisme, marquées par le retour du conservatisme en art. Lui qui n'a jamais fait acte d'allégeance au nouveau régime (ses nœuds papillons semblent une sorte de défi aux «vestes de cuir » des maîtres du jour) se trouve alors récupéré, utilisé comme un garant culturel du régime soviétique - comme en témoigne la tournée triomphale et tragique (à cause de la menace qui pesait sur chaque rencontre et lui interdisait de revoir librement ses vieux amis émigrés) qu'il fit en France en 1937, un an avant sa mort, jouant par une sorte de malentendu entretenu par Staline le rôle d'un émissaire culturel officiel de l'État soviétique.

Pendant les années 30 , il privilégie l'aspect fantaisiste, divertissant, des mises en scène, laissant de côté toute ambition novatrice. Il monte pourtant une pièce d'actualité, ou presque : Les Jours des Tourbine de Boulgakov, qui peint d'une manière nuancée, non dogmatique, les nouvelles conditions de vie créées par la révolution. À vrai dire, cette pièce est rapidement retirée de l'affiche.

51 Ses dernières années sont surtout consacrées à ses oeuvres théoriques (La Formation de l'acteur, La Construction du personnage - elles ont toutes été traduites en français) et à ses souvenirs. Ma Vie dans l'art est traduit en français en 1934 avec une préface de Jacques Copeau. Stanislavski meurt en 1938. 